Cahiers $d u$ MONDE RUSSE

\section{Cahiers du monde russe}

Russie - Empire russe - Union soviétique et États indépendants

50/2-3 | 2009

L'Europe orientale, 1650-1730. Crises, conflits et renouveau

\title{
Exotismes dans la culture russeLeonid HELLER, Anne COLDEFY-FAUCARD, éds
}

, (2-3) 283, 2009, $351 \mathrm{p}$.

Natalia Gamalova

\section{(2) OpenEdition}

Journals

\section{Édition électronique}

URL : https://journals.openedition.org/monderusse/9794

DOI : 10.4000/monderusse. 9794

ISSN : $1777-5388$

\section{Éditeur}

Éditions de l'EHESS

\section{Édition imprimée}

Date de publication : 15 septembre 2009

ISBN : 978-2-7132-2260-3

ISSN : $1252-6576$

\section{Référence électronique}

Natalia Gamalova, «Exotismes dans la culture russeLeonid HELLER, Anne COLDEFY-FAUCARD, éds », Cahiers du monde russe [En ligne], 50/2-3 | 2009, mis en ligne le 14 janvier 2013, consulté le 03 septembre 2022. URL : http://journals.openedition.org/monderusse/9794 ; DOI : https://doi.org/ 10.4000/monderusse. 9794

Ce document a été généré automatiquement le 3 septembre 2022.

Tous droits réservés 


\title{
Exotismes dans la culture russeLeonid HELLER, Anne COLDEFY-FAUCARD, éds
}

\author{
, (2-3) 283, 2009, $351 \mathrm{p}$.
}

\author{
Natalia Gamalova
}

\section{Leonid HELLER, Anne COLDEFY-FAUCARD, éds., Exotismes dans la culture russe, Lausanne : Université de Lausanne, Études de Lettres, (2-3) 283, 2009, 351 p.}

1 L'ouvrage collectif Exotismes dans la culture russe a réuni des chercheurs suisses, russes et français. Le thème, bien que reconnu dans les études de lettres, est inhabituel. Objet d'une abondante bibliographie dans la culture européenne, dans les lettres slaves, le sujet n'a guère été abordé qu'au prisme réducteur du colonialisme et de la littérature du Caucase et en de rares ouvrages de synthèse. En vingt articles, le recueil ne nous propose pas pour autant le simple déplacement de "l'invention française» sur le terrain russe: il « dialogue avec la tradition française » (L. Heller), en fait découvrir de nouvelles facettes, variant la problématique sous divers angles. Le Caucase, lieu exotique type du romantisme russe, est évoqué à travers le préceptorat de l'écrivain suisse Pierre Sciobéret et les aventures d'un Abdallah Schlatter, "Suisse d'origine et Turc de nom », dans l'article documenté et érudit de Jean-Christophe Emmenegger (Fribourg-Lyon), «Pierre Sciobéret en 1857-1864 du Moléson au Caucase, en passant par Saint-Gall et la NZZ ». Avec Daria Chemelina (Novosibirsk), nous découvrons l'architecture et les fonctions des places fortes des lignes de défense sibérienne du XVIII siècle. La rigueur d'un Vauban, implantée en Sibérie, affirme "l'européanité» de la Russie face aux autochtones turco-mongols et à l'Asie frontalière. Judicieusement, l'article ouvre ce volume plaçant le lecteur à l'intersection de l'Europe et de l'Asie, de l'architecture et de la géographie, le conduit à la frontière ${ }^{74}$, à cet espace confiné et étendu où se ressentent si intensément l'indiscrète réciprocité des regards, la méfiance, la curiosité et l'attrait. 
2 Dans sa minutieuse recherche lexicologique et sémantique, Ekaterina Velmezova (Lausanne) note : «Ce sont les plantes qui sont devenues l'un des exemples préférés des auteurs des dictionnaires russes à la recherche d'illustrations linguistiques de ce qui, en principe, pourrait être exotique... » (p. 175). Pour Igor Pilschikov (Moscou), « Les plantes exotiques odorantes chez Batiouchkov et Pouchkine : la genèse et la fonction du motif », le nard, le cinnamome, le safran évoquent bien contrées et époques : Antiquité grecque et romaine, parfums sensuels de l'Orient, Orient biblique, paradis judéo-chrétien. L'auteur démontre que l'association "nard-cinnamome» dans la traduction batiouchkovienne des élégies de Tibulle a son origine dans le Cantique des Cantiques. Au sens plus large, il examine la question du « degré d'altérité de l'étranger », de l'assimilation du lointain par le biais des cultures médiatrices proches. Andréj Dobritsyn (Lausanne) poursuit le thème de l'Orient et des textes intermédiaires, "Sagesse orientale "ad usum domesticum": Viazemski et Pouchkine ». Il étudie les nombreuses sources françaises du XVIII siècle des six fables persanes de Petr Viazemskij. En seconde partie, l'article éclaire la genèse et les transformations du motif «Et ne dispute pas avec les ignorants» chez Puškin. Cette savante enquête n'a rien à envier à un récit à énigme.

3 Dans «La franc-maçonnerie comme exotisme dans Guerre et paix», Julie Bouvard (Lausanne) introduit la notion d'« exotisme spirituel » à partir de trois éléments du roman de Tolstoj: l'égarement de Petr Bezuhov, le rite d'initiation, la fascination exercée par de mystérieuses loges. Le tournant franc-maçonnique est décrit en termes «spatiaux » d'un cheminement vers l'inconnu. Un ou deux renvois bibliographiques auraient pu éclairer sur « l'association entre le chemin spirituel et le voyage » en tant qu'« un des lieux communs les plus anciens et les plus féconds de l'imagerie chrétienne primitive » (p. 61) ; car, à première vue, c'est l'inverse du propos avancé par l'auteur qui semblerait prévaloir : le pèlerinage vers un lieu concret, les déplacements physiques et géographiques supplantent ou expriment la recherche intime du moi. Le passage sur «toute la tradition du travestissement exotique, lorsque le Blanc se pare des plumes du Nouveau Monde, du masque africain, ou encore du peignoir oriental » appelait des précisions bibliographiques. Dans Les Tsiganes de Puškin, cité subséquemment, rien ne dit qu'Aleko porte de "bariolés oripeaux", même s'il est difficile de l'imaginer en redingote. Le travestissement, le jeu vestimentaire, le bal masqué représentent une convention qui se conçoit dans un milieu habituel, qui sert à « décaler » la vie ordinaire, - ils ne relèvent donc guère d'un exotisme colonial qui suggère l'authenticité de l'être insolite, tout aussi bien que la distance entretenue par le «Blanc». Ce dernier récolte peaux et plumes pour en faire ses trophées, pour les exhiber dans son «musée personnel » (ce sera une "altérité maîtrisée, vidée de la vitalité », comme l'écrit B. Czerny dans ce même recueil, p. 91), non pour se déguiser ${ }^{75}$. Le peignoir oriental n'est pas un déguisement non plus : c'est une tenue d'intérieur bien connue en Europe, au point de devenir l'accoutrement préféré d'un Oblomov.

Originale est l'étude de Svetlana Gorshenina (Paris-Lausanne), « Cristallisation de l'image $\mathrm{du}$ Turkestan russe dans les premières expositions “coloniales” en Russie ». L'auteur montre comment la peinture participe à la construction de l'image des indigènes, ou encore la façon dont se fait le choix des échantillons représentatifs du territoire conquis. Il s'agit en particulier de l'exposition du peintre Vasilij Vereščagin à Saint-Pétersbourg et $\mathrm{du}$ pavillon turkestanais, mis en place par le naturaliste Aleksej Fedčenko, à l'Exposition Polytechnique. La " reconstitution» du Turkestan, un diorama " organisé selon les principes positivistes au goût de Linné » (p. 77) au centre de Moscou, mérite une 
attention particulière. Exprimons quelques réserves d'ordre linguistique : le terme de " particularisme " pour traduire unikal'nost' [unicité, singularité] paraît peu heureux à cause de ses connotations doctrinales ; l'expression « image médiatique » pour parler des résonances que suscite une exposition de peinture en 1869 ressemble à un anachronisme ; enfin, il y a une erreur purement "médiatique ", c'est-à-dire vulgarisée par les mass media d'aujourd'hui : "commanditaire » (p. 69) n'a rien en commun avec un zakazčik [celui qui a commandé], c'est un terme juridique qui désigne un associé qui fait un apport en capital dans une société en commandite (c'est un vkladčik, un actionnaire).

La contribution de Fanny Mossière (Lausanne), «L'exotisme du Démon: Vroubel et sa technique cristallique », s'appuie sur l'étude de la peinture. L'exotisme s'y manifeste doublement: dans l'innovation technique du peintre et dans l'orientalisme de son Démon. En effet, la nouveauté réside rarement dans les thèmes choisis par l'artiste, mais dans la manière de les traiter, dans les expérimentations avec la matière. La "juxtaposition de micro-surfaces», la fragmentation du dessin, la transposition des facettes "sculpturales» sur la toile plate, tels sont les éléments de la technique cristallique qui engendre une version singulière de l'exubérance orientale.

Boris Czerny (Caen) offre une analyse inédite de La Sauterelle de Čehov : il compare cette nouvelle aux schémas du roman colonial et met en parallèle l'exotique et le banal dans l'œuvre. L'auteur formule plusieurs concepts intéressants, dont le caractère « accumulatif» de l'exotisme; arrachés à leur contexte habituel, les trophées forment une nomenclature, et tout inventaire suppose une matérialité concentrée, exacerbée. Les pages sur la femme exotique font penser à un autre récit de Čehov - La Fange (1886) : son héroïne, Suzanne-Thamar, capture les hommes dans ses appartements aux allures d'orangerie, où règnent plantes fleuries et capiteux parfum de jasmin.

7 Une série d'articles explorent les œuvres littéraires - sources incontestables de nouvelles configurations de l'exotique. "L'exotisme en tant qu'expérience intérieure " d'Edouard Nadtotchi (Lausanne) est illustré par le roman pour enfants Le Cahier de classe et la Schwambranie de Lev Kassil'. Partant de l'idée de la conception paternaliste des colonisés, l'auteur détermine l'enfance comme une "zone coloniale», un "pays lointain», une utopie issue de l'imaginaire. Anastasia Forquenot de La Fortelle (Lausanne) analyse l'étrangeté du personnage principal du roman de Denis Gucko Un russophone: l'exotisme s'y conjugue avec la double restructuration des États postsoviétiques, avec l'hostilité des êtres qui vivent à proximité les uns des autres. La prose postsoviétique est aussi l'objet d'étude d'Hélène Mélat (Paris). Pour les héros de Dmitri Prigov, Evgenij Griškovec, Mihaïl Šiškin, Leonid Kostjukov, Vasilij Golovanov, les pays découverts après la chute du mur avaient perdu leur attrait, aussi le besoin de l'étrangeté s'est-il replié sur lui-même. Mikhaïl Nedosseïkine (Voronež) place au centre de sa réflexion Voyage au bout de la nuit de Céline. Il oppose tradition exotique orientale et tradition africaine, selon un principe que l'on pourrait résumer ainsi : passé culturel et sacré pour l'Orient, passé biologique pour l'Afrique. Ne serait-ce pas plutôt l'utilisation de ces deux exotismes dans l'art qui nous ferait distinguer, d'une part, la tradition ornementale, décorative, le luxe des Mille et Une Nuits et, de l'autre, l'art primitif de l'Afrique noire?

8 Afin de répondre à la question «que qualifiait-on et qui qualifiait-on d'exotique au début du xxe siècle », Andrej Faoustov (Voronež) sollicite la prose et les essais critiques de Gončarov, A. Grigor'ev, L. Andreev, Viač. Ivanov, Gumilev, Belyj, Brjusov, Hodasevič, Vološin, Balmont, Florenskij, etc. Sa « Sémantique de l'exotisme dans la littérature russe 
du $\mathrm{XX}^{\mathrm{e}}$ siècle » met en valeur les connotations tantôt dépréciatives, tantôt valorisantes de l'exotique : l'artificialité proche du Parnasse, l'éloignement, l'érotisme, la simplicité. Les pages sur les épices, les exotismes gastronomiques et olfactifs sont attachantes et suggestives ${ }^{76}$. La littérature de la même période est examinée dans l'article de Sergej Savinkov (Voronež) « La femme dans la littérature et la culture russes du début du $\mathrm{xx}^{\mathrm{e}}$ siècle : sujet exotique et objet d'expérience exotique ». L'auteur prolonge et enrichit les réflexions sur les balancements entre familier et étrange, entre proche et lointain; il examine en particulier les figures emblématiques d'Ève et de Lilith, l'engouement de fin de siècle pour Salomé. L'introduction de l'article d'Olga Berdnikova (Voronež), « "Tout est sauvage et splendide comme en Eden": l'œuvre d'Ivan Bounine et l'exotisme ", donne une excellente appréciation de la fonction secondaire, religieuse, mystique, philosophique de la quête des exotismes à l'žge d'argent: l'Afrique de Gumilev et de Hlebnikov, l'Égypte de Balmont, l'Inde de Rœrich. L'exotisme de l'auteur de La Rose de Jéricho, inséparable de la Bible et de la vie terrestre du Christ, poursuit et revivifie la tradition des pèlerinages vers la Terre Sainte.

Prenant à contre-pied la défamiliarisation en tant que générateur et moteur de l'exotique, Evgueni Koziouba (Voronež) met en place le concept de l'expansion de la « russité » vers les espaces exotiques dans la poésie néo-paysanne de N. Kljuev, nourrie de la tradition odique "impériale " de Lomonosov. Le Nord russe "participe » à la révélation de l'Orient contenu en son sein.

Si d'ordinaire l'exotisme rime avec pays chauds, l'espace arctique n'en est pas exclu pour autant. Bien au contraire, il permet de formuler de nouvelles interrogations : l'Arctique est-il le territoire de référence pour un écrivain russe, comment définir l'attitude du voyageur dans un univers vide, dont le paysage est vierge et dénudé? La contribution d'Anne Coldefy-Faucard (Paris), "Boris Pilniak: la tentation de l'Arctique » est l'occasion d'étendre et d'affiner l'approche des exotismes.

11 La modestie de l'intitulé de Marietta Tchoudakova (Moscou), "Quelques réflexions à propos de l'exotisme ", dissimule un texte plein d'esprit et de justesse. Puisant l'essentiel de ses modèles dans la prose soviétique des années 1920 et 1930 (Vsevolod Ivanov, Ruvim Fraerman, Vladimir Arsen'ev, Mihajl Prišvin), elle saisit les lois du fonctionnement complexe des exotismes dans la littérature et dans la société. La première partie de l'article (où l'on voit se nouer une sorte de dialogue entre l'auteur et l'éditeur, ce qui donne au texte un caractère vivant) déduit la spécificité de l'exotisme, différent de l'Autre en général, autant de la perception du sujet que de l'objet exotique lui-même. Ce dernier est inoffensif, car l'ennemi ne suscite pas de curiosité ludique. Même si nous admettons avec l'auteur que certains aspects extérieurs de l'hitlérisme ${ }^{77}$ puissent être transférés dans la sphère de l'exotique, nous proposons d'en chercher les origines dans l'officier en uniforme seyant de la culture russe. "L'esthétique des uniformes noirs", "des bottes brillantes» (p. 204) cachent moins l'ennemi qu'elles n'évoquent l'élégant officier "blanc », irrésistible face aux vareuses des "Rouges » (le mieux est encore un Rouge déguisé en Blanc comme dans le téléfilm Aide de camp de son Excellence). Au début de la guerre, Stalin abandonne l'uniforme de l'Armée rouge, trop simple, mal ajusté, balourd, pour restaurer les épaulettes et les pattes de col, pour se rapprocher de l'officier d'avant 1917, mais aussi de l'officier de la Wehrmacht. Non seulement Viačeslav Tihonov, habillé en Standartenführer, a séduit en 1973 les spectateurs et les spectatrices, mais bien avant lui Boris Barnet, Pavel Kadočnikov, Stanislav Ljubšin, Oleg Jankovskij ont incarné la beauté masculine sous l'uniforme de l'Allemagne nazie (une variante reste toujours 
préférable : l'un des nôtres travesti en officier allemand, parce que le travesti, tendu vers « l'artisticité » et la perfection, surpasse d'habitude son prototype).

Nous avons le plaisir de terminer ce long compte rendu avec la brillante contribution de Mihajl Maiatsky (Lausanne), «Comme dans le ventre de sa marâtre. Essai sur l'autoexotisation ». Non seulement l'auteur va directement au cœur du problème, mais la matière de son raisonnement nous renvoie régulièrement à notre vie contemporaine. L'exotisme devient l'angle de vue pour dire et comprendre ce que nous sommes. La simple énumération des questions intégrées au débat (norme à dépasser, omniprésence de la défamiliarisation comprise comme exotisation, recul de l'exotisme vécu au profit de l'exotisme évoqué, c'est-à-dire le phénomène culturel secondaire, auto-exotisation comme image de soi que l'on veut donner, etc.) ne saurait rendre ni la richesse intellectuelle et thématique de l'article, ni l'audacieuse verve de sa langue.

«La relation de l'altérité n'est ni spatiale, ni conceptuelle» (E. Levinas), ni temporelle non plus - elle est ontologique : «être Autre, c'est être tout court " ${ }^{78}$. L'effet exotique, quant à lui, " provient du contact ", c'est une attitude (M. Maiatsky, p. 296). La diversité des contributions destinées à éclairer ce rapport à, c'est-à-dire quelque chose qui est difficilement perceptible car dynamique, ne nuit pas à l'unité du recueil. Plusieurs définitions se répondent et se complètent, elles suscitent des réflexions qui viendront alimenter de nombreux champs de recherche. L'étude conclusive de Leonid Heller, "Décrire les exotismes: quelques propositions", est composée de sept thèmes: 1. terminologie de l'exotique, 2. histoire des exotismes, 3. théorie de l'exotique: le modernisme, 4. théorie de l'exotique : le postmodernisme, 5. classification des exotismes, 6. compagnons de route de l'exotisme (orientalisme, édénisme, utopisme, primitivisme, régionalismes), 7. exotisme et texte. Cette description (le mot est trop modeste) synthétique frôle l'exhaustivité en la matière et fait du volume non seulement un précieux ouvrage scientifique, mais aussi un véritable outil pédagogique.

\section{NOTES}

74. Le dernier article du recueil mentionnera en note marginale les Cosaques, «à la fois nôtres et autres » (p. 308); or, les Cosaques, mi-paysans, mi-guerriers, dont on ne sait pas trop si c'est une catégorie sociale spécifique ou bien une sub-ethnie, plus libres et plus rebelles que les autres, peuplaient ces zones frontalières de l'Empire russe.

75. Cette affirmation fait penser à la mode qui, à la recherche de la nouveauté, intègre régulièrement les détails des costumes exotiques, sans jamais les copier entièrement. $\mathrm{Cf}$. "Les robes à la chinoise, serrées aux hanches" au début du $\mathrm{xx}^{\mathrm{e}}$ siècle (L. LejeuneFrançoise, L. Lamourette, À la découverte de l'histoire du costume, t. III, P. : Éditions Roudil, 2001, p. 117). Paul Poiret étudia les turbans hindous au musée de Kensington. De nombreuses Parisiennes lui demandèrent de s'inspirer des maquettes «orientales » de Bakst. (P. Poiret, En habillant l'époque, P. : Grasset, 1930, p. 132-133).

76. L'évocation des ballets russes (p. 130) alimente l'idée de la mise en place artificielle (et sans doute commerciale) d'un certain produit exotique à peu près oriental, vaguement 
caucasien, somptueusement asiatique, russe pour le public, et de partout et de nulle part. En parlant, dans ce même volume, de la danse d'Ida Rubinstein dans La nuit de Cléopâtre et des costumes de Bakst, S. Savinkov (p. 169) a formulé la notion d'« exotisme provocant ", outré et forcé.

77. Les connotations sinistres et tragiques du terme, sans parler de la chose, ne le rendront jamais exotique; et l'exotique est ce domaine où le nom n'est pas inférieur à la chose, bien au contraire.

78. V.P. Litvinov, Polilogos: Problemnoe pole, Togliatti : Meždunar. akademija biznesa i banskogo dela, 1997, p. 9-10. 